\title{
State Complexity of the Set of Synchronizing Words for Circular Automata and Automata over Binary Alphabets
}

\author{
Stefan Hoffmann ${ }^{[0000-0002-7866-075 X]}$ \\ Informatikwissenschaften, FB IV, Universität Trier, Universitätsring 15, 54296 Trier, \\ Germany, hoffmanns@informatik.uni-trier.de
}

\begin{abstract}
Most slowly synchronizing automata over binary alphabets are circular, i.e., containing a letter permuting the states in a single cycle, and their set of synchronizing words has maximal state complexity, which also implies complete reachability. Here, we take a closer look at generalized circular and completely reachable automata. We derive that over a binary alphabet every completely reachable automaton must be circular, a consequence of a structural result stating that completely reachable automata over strictly less letters than states always contain permutational letters. We state sufficient conditions for the state complexity of the set of synchronizing words of a generalized circular automaton to be maximal. We apply our main criteria to the family $\mathscr{K}_{n}$ of automata that was previously only conjectured to have this property.
\end{abstract}

Keywords: finite automata $\cdot$ synchronization $\cdot$ completely reachable automata $\cdot$ state complexity $\cdot$ set of synchronizing words

\section{Introduction}

A deterministic semi-automaton is synchronizing if it admits a reset word, i.e., a word which leads to some definite state, regardless of the starting state. This notion has a wide range of applications, from software testing, circuit synthesis, communication engineering and the like, see 1517. The famous Černý conjecture [5] states that a minimal synchronizing word has length at most $(n-1)^{2}$ for an $n$ state automaton. We refer to the mentioned survey articles [15]17 for details. An automaton is completely reachable, if for each subset of states we can find a word which maps the whole state set onto this subset. This is a generalization of synchronizability, as a synchronizing word maps the whole state set to a singleton set. The class of completely reachable automata was formally introduced in [3, but already in 612] such automata appear in the results. The time complexity of deciding if a given automaton is completely reachable is unknown. A sufficient and necessary criterion for complete reachability of a given automaton in terms of graphs and their connectivity is known 4, but it is not known if these graphs could be constructed in polynomial time. A special case of the general graph construction, which gives a sufficient criterion for complete reachability [3], is known to be constructible in polynomial time [9]. The size of 
a minimal automaton accepting a given regular language is called the state complexity of that language. The set of synchronizing words of a given automaton is a regular ideal language whose state complexity is at most exponential in the size of the original automaton [1213. The Črný family of automata [5, a family of automata yielding the lower bound $(n-1)^{2}$ for the length of shortest synchronizing words, is completely reachable, but also the set of synchronizing words has maximal state complexity 1213. These properties are shared by many families of automata that are also slowly synchronizing [121213], i.e., those for which a shortest reset word is close to the Černý bound.

Outline and Contribution: In Section 2 we give definitions and state known results. Then, in Section 3 , we give a general criterion for completely reachable automata to deduce that the set of synchronizing words has maximal state complexity. We also state a structural result by which we can deduce that in completely reachable automata, where the number of letters is strictly less than the number of states, we must have permutational letters generating a non-trivial permutation group. In Section 4 we state sufficient conditions for generalized circular automata to deduce that their set of synchronizing words has maximal state complexity. In Section 5 we apply the results from Section 3 to deduce that completely reachable automata over binary alphabets must be circular and must have a letter mapping precisely two states to a single state. Also, with the results from Section 4 , we show that the family $\mathscr{K}_{n}, n>5$ odd, from 13 gives completely reachable automata such that the set of synchronizing words has maximal state complexity. This solves an open problem from [13], where this was only conjectured. The Cerný family of automata 517, the first given family yielding the lower bound $(n-1)^{2}$ for the length of synchronizing words, is completely reachable and its set of synchronizing words has maximal state complexity 1213 . These properties are also shared by a wealth of different slowly synchronizing automata 121213 . Our criteria apply to all the automata mentioned in this previous work. However, we give an example showing that our stated conditions are only sufficient, but not necessary.

\section{Preliminaries and Definitions}

General Notions: Let $\Sigma=\left\{a_{1}, \ldots, a_{k}\right\}$ be a finite set of symbols (also called letters), called an alphabet. The set $\Sigma^{*}$ denotes the set of all finite sequences, i.e., of all words or strings. The finite sequence of length zero, or the empty word, is denoted by $\varepsilon$. We set $\Sigma^{+}=\Sigma^{*} \backslash\{\varepsilon\}$. For a given word $w \in \Sigma^{*}$, we denote by $|w|$ its length. The subsets of $\Sigma^{*}$ are called languages. For $n>0$ we set $[n]=\{0, \ldots, n-1\}$ and $[0]=\varnothing$. If $a, b \in \mathbb{Z}$ and $b \neq 0$, by $a \bmod b$ we denote the unique number $0 \leqslant r<|b|$ with $a=q b+r$ for some $q \in \mathbb{Z}$. For some set $X$ by $\mathcal{P}(X)$ we denote the power set of $X$, i.e, the set of all subsets of $X$. Every function $f: X \rightarrow Y$ induces a function $\hat{f}: \mathcal{P}(X) \rightarrow \mathcal{P}(Y)$ by setting $\hat{f}(Z):=\{f(z) \mid z \in Z\}$. Here, we will denote this extension also by $f$. Let $k \geqslant 1$. A $k$-subset $Y \subseteq X$ is a finite set of cardinality $k$. 
Automata-Theoretic Notions: A finite, deterministic and complete automaton will be denoted by $\mathscr{A}=\left(\Sigma, Q, \delta, s_{0}, F\right)$ with $\delta: Q \times \Sigma \rightarrow Q$ the state transition function, $Q$ a finite set of states, $s_{0} \in Q$ the start state and $F \subseteq Q$ the set of final states. The properties of being deterministic and complete are implied by the definition of $\delta$ as a total function. The transition function $\delta: Q \times \Sigma \rightarrow Q$ could be extended to a transition function on words $\delta^{*}: Q \times \Sigma^{*} \rightarrow Q$ by setting $\delta^{*}(s, \varepsilon):=s$ and $\delta^{*}(s, w a):=\delta\left(\delta^{*}(s, w), a\right)$ for $s \in Q, a \in \Sigma$ and $w \in \Sigma^{*}$. In the remainder we drop the distinction between both functions and will also denote this extension by $\delta$. For $S \subseteq Q$ and $w \in \Sigma^{*}$, we write $\delta(S, w)=\{\delta(s, w) \mid s \in S\}$ and $\delta^{-1}(S, w)=\{q \in Q \mid \delta(q, w) \in S\}$. The language accepted by $\mathscr{A}=\left(\Sigma, S, \delta, s_{0}, F\right)$ is $L(\mathscr{A})=\left\{w \in \Sigma^{*} \mid \delta\left(s_{0}, w\right) \in F\right\}$. A language $L \subseteq \Sigma^{*}$ is called regular if $L=L(\mathscr{A})$ for some finite automaton $\mathscr{A}$. For a language $L \subseteq \Sigma^{*}$ and $u, v \in \Sigma^{*}$ we define the Nerode right-congruence with respect to $L$ by $u \equiv_{L} v$ if and only if $\forall x \in \Sigma: u x \in L \leftrightarrow v x \in L$. The equivalence class for some $w \in \Sigma^{*}$ is denoted by $[w]_{\equiv L}:=\left\{x \in \Sigma^{*} \mid x \equiv_{L} w\right\}$. A language is regular if and only if the above right-congruence has finite index, and it could be used to define the minimal deterministic automaton $\mathscr{A}_{L}=\left(\Sigma, Q, \delta,[\varepsilon]_{\equiv_{L}}, F\right)$ with $Q:=\left\{[w]_{\equiv_{L}} \mid w \in \Sigma^{*}\right\}, \delta\left([w]_{\equiv_{L}}, a\right):=[w a]_{\equiv_{L}}$ for $a \in \Sigma, w \in \Sigma^{*}$ and $F:=\left\{[w]_{\equiv_{L}} \mid w \in L\right\}$. It is indeed the smallest automaton accepting $L$ in terms of the number of states, and we will refer to this construction as the minimal automaton [11] of $L$. The state complexity of a regular language is defined as the number of Nerode right-congruence classes. We will denote this number by $\operatorname{sc}(L)$. Let $\mathscr{A}=\left(\Sigma, Q, \delta, s_{0}, F\right)$ be an automaton. A state $q \in Q$ is reachable, if $q=\delta\left(s_{0}, u\right)$ for some $u \in \Sigma^{*}$. We also say that a state $q$ is reachable from a state $q^{\prime}$ if $q=\delta\left(q^{\prime}, u\right)$ for some $u \in \Sigma^{*}$. Two states $q, q^{\prime}$ are distinguishable, if there exists $u \in \Sigma^{*}$ such that either $\delta(q, u) \in F$ and $\delta\left(q^{\prime}, u\right) \notin F$ or $\delta(q, u) \notin F$ and $\delta\left(q^{\prime}, u\right) \in F$. An automaton for a regular language is isomorphic to the minimal automaton if and only if all states are reachable and distinguishable [11. A semi-automaton $\mathscr{A}=(\Sigma, Q, \delta)$ is like an ordinary automaton, but without a distinguished start state and without a set of final states. Sometimes we will also call a semi-automaton simply an automaton if the context makes it clear what is meant. Also, definitions without explicit reference to a start state and a set of final states are also valid for semi-automata. Let $\mathscr{A}=(\Sigma, Q, \delta)$ be a finite semi-automaton. A word $w \in \Sigma^{*}$ is called synchronizing if $\delta(q, w)=\delta\left(q^{\prime}, w\right)$ for all $q, q^{\prime} \in Q$, or equivalently $|\delta(Q, w)|=1$. Set $\operatorname{Syn}(\mathscr{A})=\left\{w \in \Sigma^{*}|| \delta(Q, w) \mid=1\right\}$. The power automaton (for synchronizing words) associated to $\mathscr{A}$ is $\mathcal{P}_{\mathscr{A}}=(\Sigma, \mathcal{P}(Q), \delta, Q, F)$ with start state $Q$, final states $F=\{\{q\} \mid q \in Q\}$ and the transition function of $\mathcal{P}_{\mathscr{A}}$ is the transition function of $\mathscr{A}$, but applied to subsets of states. Then, as observed in [16], the automaton $\mathcal{P}_{\mathscr{A}}$ accepts the set of synchronizing words, i.e., $L\left(\mathcal{P}_{\mathscr{A}}\right)=\operatorname{Syn}(\mathscr{A})$. As for $\{q\} \in F$, we also have $\delta(\{q\}, x) \in F$ for each $x \in \Sigma^{*}$, the states in $F$ could all be merged to a single state to get an accepting automaton for $\operatorname{Syn}(\mathscr{A})$. Also, the empty set is not reachable from $Q$. Hence $\operatorname{sc}(\operatorname{Syn}(\mathscr{A})) \leqslant 2^{|Q|}-|Q|$ and this bound is sharp [1213. We call $\mathscr{A}$ completely reachable if for any non-empty $S \subseteq Q$ there exists a word $w \in \Sigma^{*}$ with $\delta(Q, w)=S$, i.e., in the power automaton, every state is reachable from the start state. When we say a subset of states in $\mathscr{A}$ is reachable, we mean reachability in $\mathcal{P}_{\mathscr{A}}$. The state complexity of $\operatorname{Syn}(\mathscr{A})$ is maximal, 
i.e., $\operatorname{sc}(\operatorname{Syn}(\mathscr{A}))=2^{|Q|}-|Q|$, if and only if at least one singleton subset of $Q$ and all subsets $S \subseteq Q$ with $|S| \geqslant 2$ are reachable, and all non-singleton subsets are distinguishable in $\mathcal{P}_{\mathscr{A}}$. For strongly connected automata, i.e., those for which every state is reachable from every other state, is maximal iff $\mathscr{A}$ is completely reachable and all $S \subseteq Q$ with $|S| \geqslant 2$ are distinguishable in $\mathcal{P}_{\mathscr{A}}$. A permutation on a finite set $Q$ (which here will always be the set of states of some automaton) is a bijective function, a subset of permutations closed under concatenation (and function inversion, but this is implied in the finite case) is called a permutation group. The orbit of an element from $Q$ under a given permutation group on $Q$ is the sets of all elements to which this element could be mapped by elements from the permutation group. A permutation group with a single orbit, i.e., every element could be mapped to any other, is called transitive. A semi-automaton $\mathscr{A}=(\Sigma, Q, \delta)$ is called circular, if some letter acts as a cyclic permutation on all states. This family of automata was one of the first inspected with respect to the Černý-conjecture 14, and the conjecture was finally confirmed for this family $[78$. A semi-automaton $\mathscr{A}=(\Sigma, Q, \delta)$ is called generalized circular, if some word acts as a cyclic permutation on all states ${ }^{\top}$ Let $\mathscr{A}=(\Sigma, Q, \delta)$ be an automaton and for $w \in \Sigma^{*}$ define $\delta_{w}: Q \rightarrow Q$ by $\delta_{w}(q)=\delta(q, w)$ for all $q \in Q$. Then, we can associate with $\mathscr{A}$ the transformation monoid of the automaton $\mathcal{T}_{\mathscr{A}}=\left\{\delta_{w} \mid w \in \Sigma^{*}\right\}$. The rank of a map $f: Q \rightarrow Q$ on a finite set $Q$ is the cardinality of its image. For a given automaton, seeing a word as a transformation of its state set, the rank of the word is the rank of this transformation. A permutational letter is a letter of full rank, i.e., a letter inducing a permutation on the states.

Known Results: We will need the following result from [6] to deduce complete reachability of some automata families we consider.

Proposition 1 (Don [6]). Let $\mathscr{A}=(\Sigma, Q, \delta)$ be a finite circular automaton with $n$ states, where $b$ induces a cyclic permutation of the states. Suppose we have another letter $a \in \Sigma$ of rank $n-1$ and choose $s, t \in Q$ and $0<d<|Q|$ such that $\delta(Q, a)=Q \backslash\{s\},\left|\delta^{-1}(t, a)\right|=2$ and $\delta\left(s, b^{d}\right)=t$. If $d$ and $n$ are coprime, then for every non-empty set $S \subseteq Q$ of size $k$, there exists a word $w_{S}$ of length at most $n(n-k)$ such that $\delta\left(Q, w_{S}\right)=S$.

\section{General Results on the State Complexity of $\operatorname{Syn}(\mathscr{A})$}

Our first result states that for completely reachable automata, to deduce that the set of synchronizing words has maximal state complexity, we only need to show distinguishability for those subsets of states with precisely two elements.

Lemma 2 (Hoffmann [10]). Let $\mathscr{A}=(\Sigma, Q, \delta)$ be a completely reachable semi-automaton with $n$ states. Then, $\operatorname{sc}(\operatorname{Syn}(\mathscr{A}))=2^{n}-n$ if and only if all 2 -sets of states are pairwise distinguishable in $\mathcal{P}_{\mathscr{A}}$.

\footnotetext{
1 The circular automata are a proper subfamily of the generalized circular automata, as shown by $\mathscr{A}=(\{a, b\},[3], \delta)$ with $\delta(0, a)=1, \delta(1, a)=0, \delta(2, a)=2$ and $\delta(0, b)=$ $0, \delta(1, b)=2, \delta(2, b)=1$. The word $b a$ cyclically permutes the states.
} 
With the next result we can deduce information about the structure of completely reachable automata when the alphabet, or more precisely only the number of letters of rank $n-1$, is strictly smaller than the number of states. Later, for completely reachable automata over binary alphabet, we can deduce that they must be circular and have to contain a letter of rank $n-1$. Note that we formulate it with a weaker condition than full complete reachability, merely only with reachability of subsets of size $n-1$.

Proposition 3. Let $\mathscr{A}=(\Sigma, Q, \delta)$ be a semi-automaton with $n$ states, $m$ letters of rank $n-1$ and $n>m$. Then, the following conditions are equivalent:

1. every subset of size $n-1$ is reachable,

2. there exists at least one letter of rank $n-1$ and a subset of letters generating a non-trivial permutation group such that every state is in the same orbit as some state not in the image of a rank $n-1$ letter. In particlar, we have at most $m$ orbits.

Remark 1. The condition $m<n$ cannot be omitted in Proposition 3. For example, let $\mathscr{A}=(\Sigma, Q, \delta)$ with $Q=[n]$ and $\Sigma=\left\{a_{1}, \ldots, a_{n}, b_{1}, \ldots, b_{n}\right\}$ be such that for $i \in\{1, \ldots, n\}$ we have $Q \backslash\{i\}=\delta\left(Q, a_{i}\right)$ and $a_{i}$ cyclically permutes $Q \backslash\{i\}$. Furthermore, let $b_{i}$ map some fixed state $q_{i} \in Q \backslash\{i\}$ to $\delta\left(q_{i}, a_{i}\right)$ and act as the identity transformation on the rest. Then, in $\mathscr{A}$, even when only using the alphabet $a_{1}, \ldots, a_{n}$ we reach every subset of size $n-1$. But with the additional letters, $\mathscr{A}$ is also completely reachable, as the subautomaton given by $Q \backslash\{i\}$ and only the letters $a_{i}$ and $b_{i}$ equals the Cerný-automaton, which is completely reachable [13. Hence, combining these facts gives complete reachability of $\mathscr{A}$, but we have no permutational letters at all.

With this result, we can derive that a completely reachable automaton whose alphabet is small enough has to contain a non-trivial permutation group as part of its transformation monoid. Or more specifically, if we only have a single letter of rank $n-1$, this permutation group must be transitive.

Corollary 4. If $\mathscr{A}=(\Sigma, Q, \delta)$ is completely reachable with only a single nonpermutational letter and $|Q|>2$, then $\mathcal{T}_{\mathscr{A}}$ contains a transitive permutation group as a submonoid.

If we find a transitive permutation group in the transformation monoid of some given automaton, then this automaton is strongly connected. Hence, let us state the following observation concerning strongly connected automata.

Lemma 5 (Hoffmann [10]). Let $\mathscr{A}=(\Sigma, Q, \delta)$ be strongly connected. If $\operatorname{Syn}(\mathscr{A})$ has maximal state complexity, then $\mathscr{A}$ is completely reachable.

Combining Corollary 4 and Lemma 5 gives the next lemma.

Lemma 6. Let $\mathscr{A}=(\Sigma, Q, \delta)$ be completely reachable with only a single nonpermutational letter and $|Q|>2$. Then, if $\operatorname{Syn}(\mathscr{A})$ has maximal state complexity, the semi-automaton is completely reachable.

As circular automata are strongly connected, the next follows by Lemma 5 .

Corollary 7. Let $\mathscr{A}=(\Sigma, Q, \delta)$ be a circular semi-automaton. If $\operatorname{sc}(\operatorname{Syn}(\mathscr{A}))=$ $2^{n}-n$, then $\mathscr{A}$ is completely reachable. 


\section{Generalized Circular Automata}

Here, Theorem 8 and Proposition 10 give sufficient conditions to deduce, for completely reachable circular automata, that the set of synchronizing words has maximal state complexity. Both conditions entail all known cases of automata over a binary alphabets for which the set of synchronizing words has maximal state complexity [12]13. However, at the end of this section, we will show that the stated conditions are not necessary. In Theorem 8, we do not assume the automaton to be completely reachable, but only to be circular and to have a letter of rank $n-1$ fulfilling a certain condition. If we also suppose complete reachability, then the theorem gives that the set of synchronizing words has maximal state complexity. Also, note that instead of a letter, any word fulfilling the mentioned condition in Theorem 8 will work to give the conclusion. Most of the time, we formulate our results for letters, but in all statements the assumptions could be formulated with words instead, as the notions of distinguishablity do not depend on the length, but only on the existence of certain word\$2 However, we have a slight focus on automata over binary alphabets later on, and the results of Section 5 will show that completely reachable automata over binary alphabets with at least three states are always circular and every word that cyclically permutes the states is a power of the cyclic permutation. So, we formulate our result with letters instead of words for simplicity. Intuitively, in Theorem 8, Equation (1) says that we can apply the letter $a$ to reduce the distance modulo $n$ on the cycle given by $b$, or, by Equation (2), that we can map to a state having a specific distance, from which we can then reduce it. Please see Figure 1 for a graphical depiction.

Theorem 8. Suppose $\mathscr{A}=(\Sigma, Q, \delta)$ has $n$ states. Let $\{a, b\} \subseteq \Sigma$ (or any two words in $\left.\Sigma^{*}\right)$. Assume the letter $b$ cyclically permutes the states and the letter a has rank $n-1$. Then all 2 -sets are distinguishable in $\mathcal{P}_{\mathscr{A}}$, if we can find a state $q \in Q$ and a number $d>0$ coprime to $n$ such that for each $0<m<n$ w $\oint^{3}$ either have

$$
\delta\left(q, b^{m} a\right)=\delta\left(q, a b^{n+m-d}\right)
$$

or, but only in case $m$ is not divisible by $d$,

$$
\delta\left(q, a b^{r}\right)=\delta\left(q, b^{m} a\right) \text { or } \delta\left(q, b^{m} a b^{r}\right)=\delta(q, a)
$$

for some number $0 \leqslant r<n$ divisible by $d$.

\footnotetext{
${ }^{2}$ For, if we choose a finite number of words and build the automaton by identifiying these words with new letters, distinguishability or reachability of states (or subsets of states) of this new automaton is inherited to the original automaton. Hence, all results are also valid when stated with words instead of letters, but otherwise the same conditions.

${ }^{3}$ Note that $0<m<n$ implies $\delta\left(q, b^{m}\right) \neq q$. Also note that we added $n$ on the right hand side to account for values $d>1$. In Proposition 10 we only subtract one from the exponent of $b$, which is always non-zero and strictly smaller than $n$, and so we do not needed this "correction for the $b$-cycle" in case of resulting negative exponents.
} 


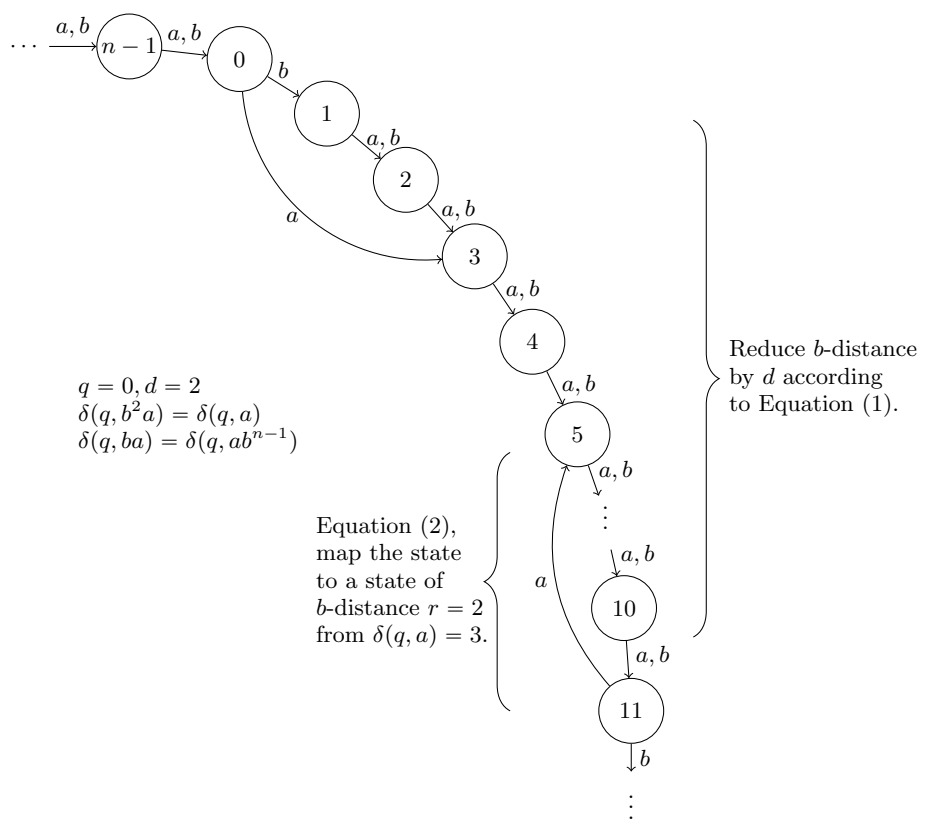

Fig. 1. Illustration of the conditions stated in Theorem 8 for an instance with $d=2$. Shown are the first twelve states and the state $n-1$ for a circular automaton with $n$ states. Note that in Theorem 8, we suppose $0<m<n$, and indeed, for $m \in\{0, n\}$ Equation 11 does not apply in general.

In the formulation of Theorem 8 , we have $r>0$, as in this case $m \neq 0$. Also, note that $\delta\left(q, b^{m} a b^{r}\right)=\delta(q, a)$ is equivalent with $\delta\left(q, a b^{n-r}\right)=\delta\left(q, b^{m} a\right)$, as for any states $s, t \in Q$ and $0 \leqslant k<n$ we have $\delta\left(s, b^{k}\right)=t$ if any only if $\delta\left(t, b^{n-k}\right)=s$, as $\delta\left(s, b^{n}\right)=s$. The conditions mentioned in Theorem 8 are the most general ones in this paper, but let us state next, as a corollary, a more relaxed formulation, stating that we can reduce the distance on the cycle by one for each application of some word of rank $n-1$.

Corollary 9. Let $\mathscr{A}=(\Sigma, Q, \delta)$ be a circular automaton with $n$ states where the letter $b$ permutes the states with a single orbit. Suppose we find a word $w \in \Sigma^{*}$ and state $q \in Q$ such that, for $0 \leqslant m<n$,

$$
\delta\left(q, b^{m+1} w\right)=\delta\left(q, w b^{m}\right)
$$

Then all 2-sets are distinguishable in $\mathcal{P}_{\mathscr{A}}$. In particular, if $\mathscr{A}$ is completely reachable, then $\operatorname{sc}(\operatorname{Syn}(\mathscr{A}))=2^{n}-n$.

Proof. Set $s=\delta(q, b)$ and $t=\delta(q, w)$. Then, $\delta(s, w)=t=\delta(q, w)$ and $s \neq q$. For $m \in\{1, \ldots, n-1\}$ we have $\delta\left(q, b^{m} w\right)=\delta\left(t, b^{m-1}\right)$ and

$$
\left\{\delta(q, b), \delta\left(q, b^{2}\right), \ldots, \delta\left(q, b^{n-1}\right)\right\}=Q \backslash\{q\} .
$$

\footnotetext{
${ }^{4}$ Note that here, even if the bounds for $m$ from Theorem 8 do not include this case, $\delta(q, w)=\delta\left(q, b^{n} w\right)=\delta\left(q, w b^{n-1}\right)$, which is equivalent with $\delta(q, w)=\delta(q, b)$.
} 
So, as $b$ is a permutation, $w$ acts injective on $Q \backslash\{q\}$ and has rank $n-1$. Now, apply Theorem 8, interpreting $w$ as the letter $a$ of rank $n-1$.

Actually, for the relaxed condition mentioned in Corollary 9 we can give a small strengthening by only requiring that we can reduce the "cyclic distance" for all states which are no more than $\lfloor n / 2\rfloor+1$ steps, or applications of $b$, away from some specific state.

Proposition 10. Let $\Sigma=\{a, b\}$ and suppose $\mathscr{A}=(\Sigma, Q, \delta)$ has $n$ states and is completely reachable with the letter a having rank $n-1$ and the letter $b$ permuting the states with a single orbit. Then $\operatorname{sc}(\operatorname{Syn}(\mathscr{A}))=2^{n}-n$ if we can find a state $q \in Q$ such that for all $0 \leqslant m \leqslant\lfloor n / 2\rfloor-1$ we have

$$
\delta\left(q, b^{m+1} a\right)=\delta\left(q, a b^{m}\right) .
$$

Finally, we show that the mentioned sufficient conditions are not necessary. In Example 1 we will give a circular automaton whose set of synchronizing words has maximal state complexity but for which this could not be derived with any of the results stated here.

Example 1. Let $\mathscr{A}=(\Sigma,[4], \delta)$ with $\Sigma=\{a, b\}, \delta(i, b)=(i+1) \bmod 4$ and $\delta(0, a)=1, \delta(1, a)=2, \delta(2, a)=1, \delta(3, a)=3$. Please see Figure 2 for a graphical depiction of $\mathscr{A}$ and $\mathcal{P}_{\mathscr{A}}$. Then, all words of rank 3 are listed in Table 1 . In each word $w$ of rank 3 the distance of the two distinct states mapped to one state is 2. So, in Equation (1), for each such word of rank 3 (in place of $a$ ), we would have $d=2$. But 2 is not coprime to 4 , hence Theorem 8 does not apply here. However, we have $\operatorname{sc}(\operatorname{Syn}(\mathscr{A}))=2^{4}-4=12$. We see in Figure 2 that every subset is reachable. We also see that $a$ distinguishes $\{0,2\}$ from every other 2set of states, $b a$ distinguishes $\{1,3\}$ from every other, baba distinguishes $\{2,3\}$ from $\{0,3\},\{1,2\}$ and $\{0,1\}$ and these latter three 2-sets are easily seen to be distinguishable by words in $b^{*} a b a$. So, by Lemma 2 all non-empty subsets of states are distinguishable.

\section{Automata over Binary Alphabets}

Here, we take a closer look at automata over a binary alphabet. We apply our results and solve an open problem posed in [13. In general, if a letter has rank $k$ and some subset is mapped to a subset of size $k$, we must hit the full image of this letter. This gives, if we only have two letters but more than two states and no letter has full rank, that we can only reach at most two subsets of size $n-1$. So, if more $(n-1)$-sets are reachable, we must have precisely one letter of rank $n-1$ and Corollary 4 gives the next result.

Lemma 11. Let $\Sigma=\{a, b\}$ be a binary alphabet and $\mathscr{A}=(\Sigma, Q, \delta)$ a finite semi-automaton with $n>2$ states. Then, the following conditions are equivalent:

1. every subset of size $n-1$ is reachable,

2. exactly one letter acts as a cyclic permutation with a single orbit and the other letter has rank $n-1$. 


\begin{tabular}{|cc|cc|cc|cc|}
\hline Word & Mapping & Word Mapping & Word & Mapping & Word & Mapping \\
\hline$b^{2} a^{2} b^{2}$ & {$[0,1,0,3]$} & $a$ & {$[1,2,1,3]$} & $a b^{3}$ & {$[0,1,0,2]$} & $a b^{2} a b$ & {$[0,2,0,3]$} \\
$a^{2} b^{2}$ & {$[0,3,0,1]$} & $b^{2} a$ & {$[1,3,1,2]$} & $b^{2} a b^{3}$ & {$[0,2,0,1]$} & $a b^{2} a^{2} b$ & {$[0,3,0,2]$} \\
$a b^{2} a^{2} b^{2}$ & {$[1,0,1,3]$} & $a^{2}$ & {$[2,1,2,3]$} & $a^{2} b^{3}$ & {$[1,0,1,2]$} & $b^{2} a b$ & {$[2,0,2,3]$} \\
$a b^{2} a b^{2}$ & {$[1,3,1,0]$} & $b^{2} a^{2}$ & {$[2,3,2,1]$} & $b^{2} a^{2} b^{3}$ & {$[1,2,1,0]$} & $a b$ & {$[2,3,2,0]$} \\
$a b^{2}$ & {$[3,0,3,1]$} & $a b^{2} a$ & {$[3,1,3,2]$} & $a b^{2} a b^{2}$ & {$[2,0,2,1]$} & $b^{2} a^{2} b$ & {$[3,0,3,2]$} \\
$b^{2} a b^{2}$ & {$[3,1,3,0]$} & $a b^{2} a^{2}$ & {$[2,2,3,1]$} & $a b^{2} a^{2} b^{3}$ & {$[2,1,2,0]$} & $a^{2} b$ & {$[3,2,3,0]$} \\
\hline$b a b^{2} a^{2} b^{2}[0,1,3,1]$ & $b a^{2}$ & {$[1,2,3,2]$} & $b a^{2} b^{3}$ & {$[0,1,2,1]$} & $b^{3} a b$ & {$[0,2,3,2]$} \\
$b a b^{2}$ & {$[0,3,1,3]$} & $b a b^{2} a$ & {$[1,2,3,2]$} & $b a b^{2} a b^{3}$ & {$[0,2,1,2]$} & $b^{3} a^{2} b$ & {$[0,3,2,3]$} \\
$b^{3} a^{2} b^{2}$ & {$[1,0,3,0]$} & $b a$ & {$[2,1,3,1]$} & $b a b^{3}$ & {$[1,0,2,0]$} & $b a b^{2} a b$ & {$[2,0,3,1]$} \\
$b^{3} a b^{2}$ & {$[1,3,0,3]$} & $b a b^{2} a^{2}$ & {$[2,3,1,3]$} & $b a b^{2} a^{2} b^{3}[1,2,0,2]$ & $b a^{2} b$ & {$[2,3,0,3]$} \\
$b a^{2} b^{2}$ & {$[3,0,1,0]$} & $b^{3} a$ & {$[3,1,2,1]$} & $b^{3} a b^{3}$ & {$[2,0,1,0]$} & $b a b^{2} a^{2} b[3,0,2,1]$ \\
$b a b^{2} a b^{2}$ & {$[3,1,0,1]$} & $b^{3} a^{2}$ & {$[3,2,1,3]$} & $b^{3} a^{2} b^{3}$ & {$[2,1,0,2]$} & $b a b$ & {$[3,2,0,2]$} \\
\hline
\end{tabular}

Table 1. All rank 3 words for the automaton from Example 1 . To the right of each word the induced transformation on the states is written, where $j \in[4]$ written at position $i \in[4]$ means the word maps the state $i$ to state $j$. The entries are ordered such that for two words $u, v$ in the same row we have $\delta(i, u)=\delta(j, u)$ iff $\delta(i, v)=\delta(j, v)$ for $i \in[4]$ and the images of words in the same column are equal.

In particular, over a binary alphabets, completely reachable automata and those whose set of synchronizing words has maximal state complexity are circular.

Remark 2. In Lemma 11, we need $n>2$. For let $\mathscr{A}=(\{a, b\},\{p, q\}, \delta)$ with $\delta(p, a)=$ $\delta(q, a)=q$ and $\delta(p, b)=\delta(q, b)=p$. Then $\mathscr{A}$ is completely reachable, but no letter acts as a non-trivial permutation.

With Theorem 8 , we can solve an open problem from [13. For $n>5$, define the automata $\mathscr{K}_{n}=(\Sigma,[n], \delta)$, introduced in [13, with

$$
\begin{aligned}
& \delta(i, b)=i+1 \text { for } i \in\{0, \ldots, n-2\}, \text { and } \delta(n-1, b)=0 \\
& \delta(i, a)=i+1 \text { for } i \in\{1, \ldots, n-3\}, \delta(n-1, a)=0, \delta(n-2, a)=1, \delta(0, a)=3 .
\end{aligned}
$$

Please see Example 2 for an illustration of this automata family. In [13, it was conjectured that $\operatorname{sc}\left(\operatorname{Syn}\left(\mathscr{K}_{n}\right)\right)=2^{n}-n$ for every odd $n>5$. With Theorem 8 , together with Proposition 1 and Lemma 2, we can confirm this.

Proposition 12. Let $n>5$ be odd. Then we have $\operatorname{sc}\left(\operatorname{Syn}\left(\mathscr{K}_{n}\right)\right)=2^{n}-n$.

Proof. First, we will show, using Proposition 1, that the automata $\mathscr{K}_{n}$, for odd $n>5$, are completely reachable. Then, we will show, using Proposition 8 , that all 2-subsets of states are distinguishable in the power automaton $\mathcal{P}_{\mathscr{K}_{n}}$. With Lemma 2, this would then give $\operatorname{sc}\left(\operatorname{Syn}\left(\mathscr{K}_{n}\right)\right)=2^{n}-n$.

1. For $n>5$ odd, the automata $\mathscr{K}_{n}$ are completely reachable: We have two letters, the letter $a$ has rank $n-1$ and the letter $b$ is a cyclic permutation of all the states. Also $\delta(Q, a)=Q \backslash\{n-1\}, \delta^{-1}(3, a)=\{0,2\}$ and $\delta\left(n-1, b^{4}\right)=3$. If $n$ is odd, then $n$ and 4 are coprime. We have listed the prerequisites of Proposition 1, hence applying it gives that $\mathscr{K}_{n}$ is completely reachable.

\footnotetext{
${ }^{5}$ I slightly changed the numbering of the states with respect to the action of the letter a compared to [13.
} 


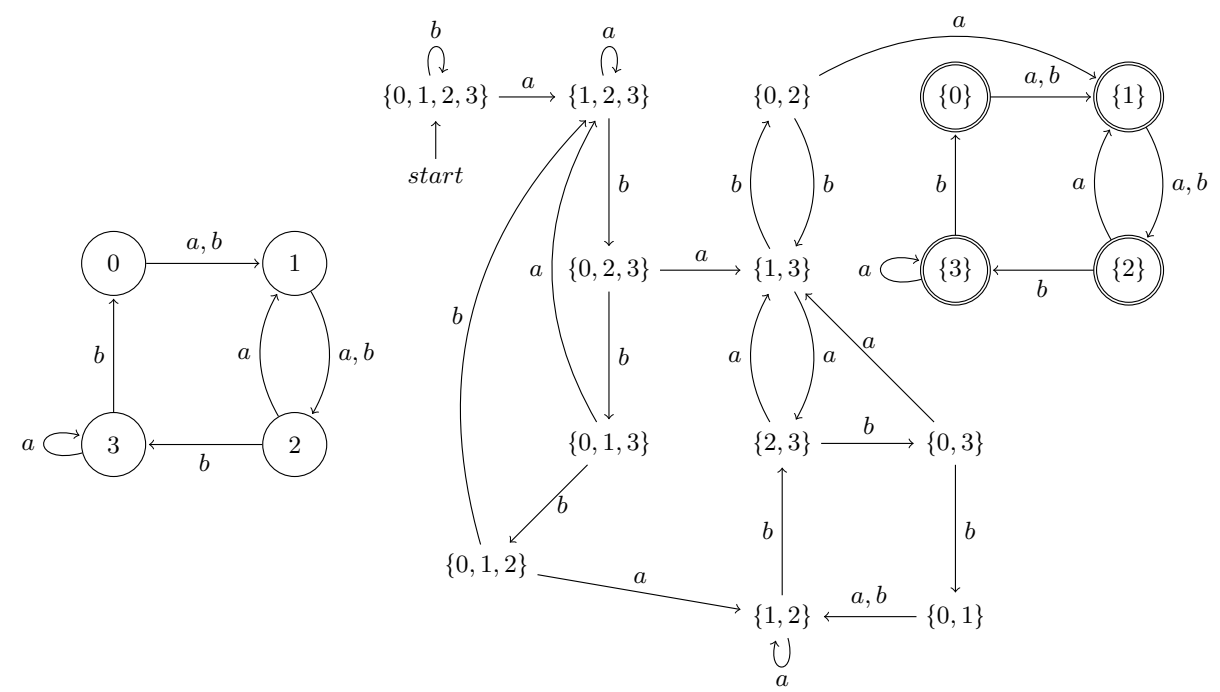

Fig. 2. The automaton from Example 1 and its power automaton. An example of an automaton whose set of synchronizing words has maximal state complexity but for which Theorem 8 or Proposition 10 do not apply, not for $a$ and not for any word of rank 3. The final states in the power automaton are marked with double circles.

2. For $n>5$ odd, in $\mathscr{K}_{n}$ all 2-sets are distinguishable in $\mathcal{P}_{\mathscr{K}_{n}}$ : Let $q=0$. Then

$$
\delta(q, b a)=2=\delta\left(q, a b^{n-1}\right)=\delta\left(q, a b^{n+1-2}\right) .
$$

For $m \in\{2, \ldots, n-3\}$, we have $\delta\left(0, b^{m}\right)=m$ and

$$
\delta\left(q, b^{m} a\right)=m+1=\delta\left(3, b^{m-2}\right)=\delta\left(q, a b^{m-2}\right)=\delta\left(q, a b^{n+m-2}\right) .
$$

The value $m=n-2$ does not follow the above pattern, but we have $\delta\left(q, b^{n-2} a b^{2}\right)=\delta\left(1, b^{2}\right)=3=\delta(q, a)$. And lastly, for $m=n-1$, we have

$$
\delta\left(q, b^{n-1} a\right)=q=\delta\left(q, a b^{n-1-2}\right) .
$$

So, with $d=2$ and $r=2$, for odd $n$, as then $n-2$ is not divisible by $d$, and with $q=0$, the prerequisites of Theorem 8 are fulfilled and give the claim.

So, both statements together with Lemma 2 yield $\operatorname{sc}\left(\operatorname{Syn}\left(\mathscr{K}_{n}\right)\right)=2^{n}-n$.

Lastly, let us give some additional examples from the literature [1|2[12]13] for which our results apply.

Example 2. Please see Figure 3 for the automata families. The automata $\mathscr{C}_{n}$ gives the Cerný family, the automata $\mathscr{L}_{n}, \mathscr{V}_{n}, \mathscr{F}_{n}$ and $\mathscr{K}_{n}$ were introduced in [1]12]13. There, except for $\mathscr{K}_{n}$, it was established that in each case (for $\mathscr{F}_{n}$ only if $n$ is odd and $n>3$ ) the set of synchronizing words has maximal state complexity. Note that our results, namely Theorem 8 together with Proposition 1 and Lemma 2 also give these results. 

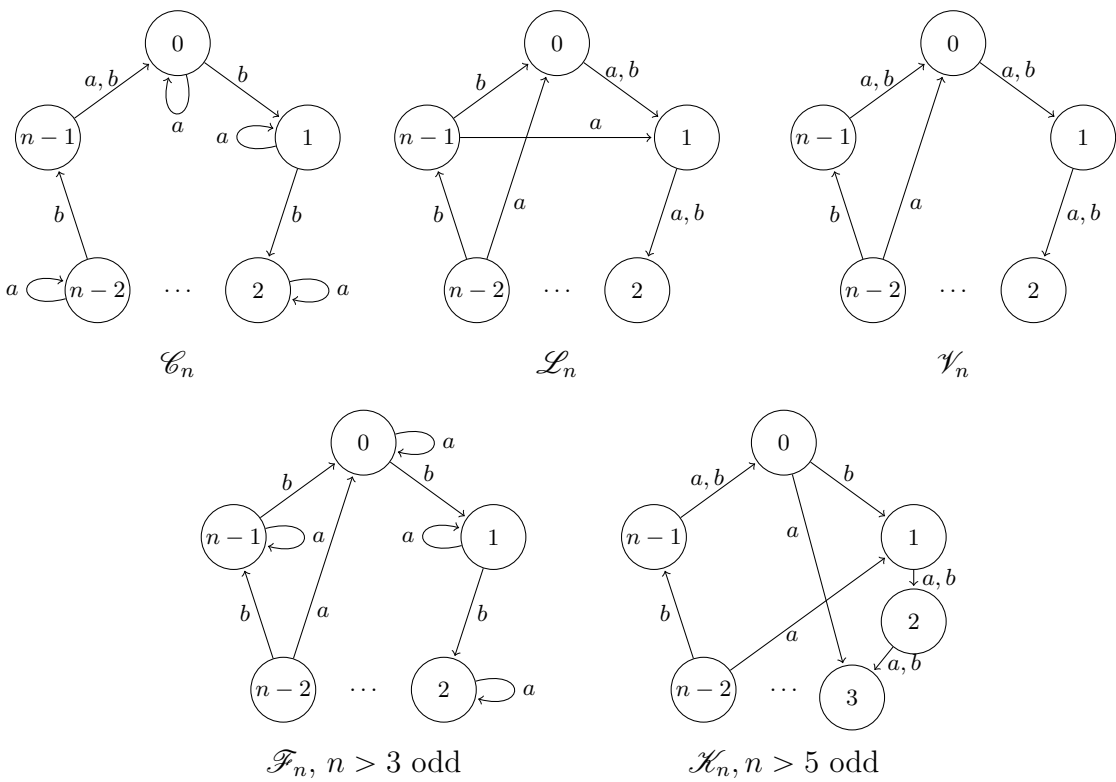

Fig. 3. Families of automata whose sets of synchronizing words have maximal state complexity. Please see Example 2 for explanation.

\section{Conclusion}

We have stated sufficient criteria for completely reachable generalized circular automata with a letter of rank $n-1$ to deduce that their set of synchronizing words has maximal state complexity. Note that by our results, every completely reachable automaton over a binary alphabet must have this form. It is natural to ask if we can generalize this to obtain a sufficient and necessary criterion. As a step in this direction, another family for which this might be tackled first is the family of circular automata $\mathscr{A}=(\Sigma, Q, \delta)$ over a binary alphabet with a rank $n-1$ letter $a$ such that $\delta(Q, a a)=\delta(Q, a)$, i.e., the set $\delta(Q, a)$ is permuted by $a$. For these automata, we can find a power of $a$ such that $a$ acts as the identity on $\delta(Q, a)$ and the single state in $Q \backslash \delta(Q, a)$ is mapped into $\delta(Q, a)$. Note that these automata closely resemble those of the Črný family. Hence, we can find easy sufficient criteria for these automata by applying our obtained results to the resulting automaton, where the power of $a$ is considered as the new rank $n-1$ letter. However, as Example 1] shows, such a criterion is also not necessary. But a more finer analysis might give sufficient and necessary criteria.

\section{References}

1. Ananichev, D.S., Gusev, V.V., Volkov, M.V.: Slowly synchronizing automata and digraphs. In: Hlinený, P., Kucera, A. (eds.) Mathematical Foundations of Computer Science 2010, 35th International Symposium, MFCS 2010, Brno, Czech Republic, 
August 23-27, 2010. Proceedings. Lecture Notes in Computer Science, vol. 6281, pp. 55-65. Springer (2010)

2. Ananichev, D.S., Volkov, M.V., Gusev, V.V.: Primitive digraphs with large exponents and slowly synchronizing automata. Journal of Mathematical Sciences 192(3), 263-278 (2013)

3. Bondar, E.A., Volkov, M.V.: Completely reachable automata. In: Câmpeanu, C., Manea, F., Shallit, J. (eds.) Descriptional Complexity of Formal Systems - 18th IFIP WG 1.2 International Conference, DCFS 2016, Bucharest, Romania, July 5-8, 2016. Proceedings. Lecture Notes in Computer Science, vol. 9777, pp. 1-17. Springer (2016)

4. Bondar, E.A., Volkov, M.V.: A characterization of completely reachable automata. In: Hoshi, M., Seki, S. (eds.) Developments in Language Theory - 22nd International Conference, DLT 2018, Tokyo, Japan, September 10-14, 2018, Proceedings. Lecture Notes in Computer Science, vol. 11088, pp. 145-155. Springer (2018)

5. Černý, J.: Poznámka k homogénnym experimentom s konečnými automatmi. Matematicko-fyzikálny časopis 14(3), 208-216 (1964)

6. Don, H.: The Černý conjecture and 1-contracting automata. Electr. J. Comb. 23(3), P3.12 (2016)

7. Dubuc, L.: Les automates circulaires biaisés vérifient la conjecture de Cerný. ITA 30(6), 495-505 (1996)

8. Dubuc, L.: Sur les automates circulaires et la conjecture de Cerný. ITA 32(1-3), 21-34 (1998)

9. Gonze, F., Jungers, R.M.: Hardly reachable subsets and completely reachable automata with 1-deficient words. Journal of Automata, Languages and Combinatorics 24(2-4), 321-342 (2019)

10. Hoffmann, S.: Completely reachable automata, primitive groups and the state complexity of the set of synchronizing wordsng. In: Language and Automata Theory and Applications - 15th International Conference, LATA 2021, Milan, Italy, March 1-5, 2021, Proceedings. Lecture Notes in Computer Science, Springer (2021)

11. Hopcroft, J.E., Ullman, J.D.: Introduction to Automata Theory, Languages, and Computation. Addison-Wesley Publishing Company (1979)

12. Maslennikova, M.I.: Reset complexity of ideal languages. CoRR abs/1404.2816 (2014)

13. Maslennikova, M.I.: Reset complexity of ideal languages over a binary alphabet. Int. J. Found. Comput. Sci. 30(6-7), 1177-1196 (2019)

14. Pin, J.: Sur un cas particulier de la conjecture de Cerny. In: Ausiello, G., Böhm, C. (eds.) Automata, Languages and Programming, Fifth Colloquium, Udine, Italy, July 17-21, 1978, Proceedings. Lecture Notes in Computer Science, vol. 62, pp. 345-352. Springer (1978)

15. Sandberg, S.: Homing and synchronizing sequences. In: Broy, M., Jonsson, B., Katoen, J.P., Leucker, M., Pretschner, A. (eds.) Model-Based Testing of Reactive Systems. LNCS, vol. 3472, pp. 5-33. Springer (2005)

16. Starke, P.H.: Eine Bemerkung über homogene Experimente. Elektronische Informationsverarbeitung und Kybernetik (later Journal of Information Processing and Cybernetics) 2(4), 257-259 (1966)

17. Volkov, M.V.: Synchronizing automata and the Černý conjecture. In: Martín-Vide, C., Otto, F., Fernau, H. (eds.) Language and Automata Theory and Applications, 2nd Int. Conference, LATA. LNCS, vol. 5196, pp. 11-27. Springer (2008) 


\section{A Proofs for Section 3 (General Results on the State Complexity of $\operatorname{Syn}(\mathscr{A})$ )}

Proposition 3. Let $\mathscr{A}=(\Sigma, Q, \delta)$ be a semi-automaton with $n$ states, $m$ letters of rank $n-1$ and $n>m$. Then, the following conditions are equivalent:

1. every subset of size $n-1$ is reachable,

2. there exists at least one letter of rank $n-1$ and a subset of letters generating a non-trivial permutation group such that every state is in the same orbit as some state not in the image of a rank $n-1$ letter. In particlar, we have at most $m$ orbits.

Proof. Let us denote the rank of a function $f:[n] \rightarrow[n]$ by $\operatorname{rk}(f)$. Note that

$$
\operatorname{rk}(f g) \leqslant \min \{\operatorname{rk}(f), \operatorname{rk}(g)\}
$$

for functions $f, g:[n] \rightarrow[n]$. Set $n=|Q|$. First, assume that every subset of size $n-1$ is reachable. Then some letter, say $a_{1}$, must have rank $|Q|-1$. For, if not, then any word $w \in \Sigma^{*}$ has rank $|Q|$, if it is composed out of permutations, or a rank strictly smaller than $|Q|-1$, if some letter of rank strictly smaller than $|Q|-1$ appears in it, by Equation (5). Let $a_{1}, \ldots, a_{m}$ be all those letters of rank $n-1$ and let $G$ be the subgroup generated by all permutational letters. We will employ a slight abuse of notation in the following, we will write, for a state $q \in Q$ and $g \in G$, simply $\delta(q, g)$ when we actually mean to choose a word $w$ representing $g$ and applying this to $q$, i.e., $\delta(q, w)$. This poses no problem and is well-defined, as $G$ is defined as a subset of the transformation monoid, which identifies words precisely if they define the same transformation. Choose, for each $i \in\{1, \ldots, m\}$, a state $s_{i} \notin \delta\left(Q, a_{i}\right)$, i.e., $Q=\delta\left(Q, a_{i}\right) \cup\left\{s_{i}\right\}$. Note that, for $A \subseteq Q$, if $\left|\delta\left(A, a_{i}\right)\right|=n-1, i \in\{1, \ldots, m\}$, then $\delta\left(A, a_{i}\right)=\delta\left(Q, a_{i}\right)$. This yields that for any reachable subset $B \subseteq Q$ of size $n-1$ we could write $B=\delta\left(Q, a_{i} g\right)$ with $g \in G$ and some $i \in\{1, \ldots, m\}$. Hence, $B=Q \backslash\left\{\delta\left(s_{i}, g\right)\right\}$. Let $q \in Q$. Then $Q \backslash\{q\}$ is reachable by assumption. Hence, as the elements in $G$ are permutations, $q=\delta\left(s_{i}, g\right)$ for some $g \in G$. This yields

$$
Q=\delta\left(s_{1}, G\right) \cup \ldots \cup \delta\left(s_{m}, G\right)
$$

If $G$ is trivial, then $\delta\left(s_{1}, G\right)=\left\{s_{1}\right\}$. So, as $n>m, G$ must be non-trivial, i.e., we must have some letter of full rank.

Now, conversely assume the letters of rank $n-1$ are $a_{1}, \ldots, a_{m}$ for some $m>0$ with $\delta\left(Q, a_{i}\right) \cup\left\{s_{i}\right\}=Q$ for states $s_{i} \in Q, i \in\{1, \ldots, m\}$ such that

$$
Q=\delta\left(s_{1}, G\right) \cup \ldots \cup \delta\left(s_{m}, G\right) .
$$

Choose some $q \in Q$ and suppose $q=\delta\left(s_{i}, g\right)$ for $i \in\{1, \ldots, m\}$ and $g \in G$. Then, as $g$ is a permutation, $Q \backslash\{q\}=\delta\left(Q \backslash\left\{s_{i}\right\}, g\right)$ and as $Q \backslash\left\{s_{i}\right\}=\delta\left(Q, a_{i}\right)$ we find $Q \backslash\{q\}=\delta\left(Q, a_{i} g\right)$ 


\section{B Proofs for Section 4 (Generalized Circular Automata)}

Theorem 8. Suppose $\mathscr{A}=(\Sigma, Q, \delta)$ has $n$ states. Let $\{a, b\} \subseteq \Sigma$ (or any two words in $\Sigma^{*}$ ). Assume the letter $b$ cyclically permutes the states and the letter a has rank $n-1$. Then all 2 -sets are distinguishable in $\mathcal{P}_{\mathscr{A}}$, if we can find a state $q \in Q$ and a number $d>0$ coprime to $n$ such that for each $0<m<n$ w $€^{6}$ either have

$$
\delta\left(q, b^{m} a\right)=\delta\left(q, a b^{n+m-d}\right)
$$

or, but only in case $m$ is not divisible by $d$,

$$
\delta\left(q, a b^{r}\right)=\delta\left(q, b^{m} a\right) \text { or } \delta\left(q, b^{m} a b^{r}\right)=\delta(q, a)
$$

for some number $0 \leqslant r<n$ divisible by $d$.

Proof. Choose the notation for $q \in Q$ and $r, d>0$ as in the statement. Suppose $n>1$. Note that $\delta\left(q, b^{d} a\right)=\delta(q, a)$, where $\delta\left(q, b^{d}\right) \neq q$, as $d$ is coprime to $n$. Hence, as $a$ has rank $n-1$, it is injective on $Q \backslash\{q\}$. Choose $0 \leqslant s<n$ such that $\delta(q, a)=\delta\left(q, b^{s}\right)$. Then note that, as $\delta\left(q, b^{m} a\right)=\delta\left(q, b^{s+n+m-d}\right)$ or $\delta\left(q, b^{m} a\right)=\delta\left(q, b^{s+r}\right)$ or $\delta\left(q, b^{m} a\right)=\delta\left(q, b^{s+n-r}\right)$, the set of these equations together with the state $\delta(q, a)$ completely determine the action of the letter $a$.

Let $\left\{p_{0}, q_{0}\right\},\left\{s_{0}, t_{0}\right\} \subseteq Q$ be two distinct 2 -sets. We want to show that we can distinguish them in $\mathcal{P}_{\mathscr{A}}$. First, let $u \in b^{*}$ be such that $\delta\left(q_{0}, u\right)=q$. By applying $u$ to both subsets of $Q$, we can assume that $q_{0}=q$ with respect to distinguishability. Write $p_{0}=\delta\left(q_{0}, b^{m_{0}}\right)$ for some $0<m_{0}<n$. Define, for $i \geqslant 0$, the sequences $\left\{p_{i}\right\},\left\{q_{i}\right\},\left\{s_{i}\right\}$ and $\left\{t_{i}\right\}$ by

$$
x_{i+1}=\delta\left(x_{i}, a b^{n-s}\right), \quad x \in\{p, q, s, t\}
$$

where $p_{0}, q_{o}, s_{0}, t_{0}$ are given as above. Also set $0 \leqslant m_{i}<n$ with $\delta\left(q_{i}, b^{m_{i}}\right)=p_{i}$. Then, we have the following.

Claim 1: For all $i \geqslant 0$ we have $q_{i}=q$.

Proof of Claim 1. It is $q_{0}=q$ by definition. Then, inductively assuming $q_{i}=q$, as $\delta(q, a)=\delta\left(q, b^{s}\right)$, we have $q_{i+1}=\delta\left(q_{i}, a b^{n-s}\right)=\delta\left(q, a b^{n-s}\right)=$ $\delta\left(q, b^{n}\right)=q$.

Claim 2: Let $i \geqslant 0$ and suppose $\left|\left\{p_{i}, q_{i}\right\}\right|=\left|\left\{s_{i}, t_{i}\right\}\right|=2$. If $p_{i+1}=q_{i+1}$, then $s_{i} \neq t_{i}$; and if $s_{i+1}=t_{i+1}$, then $p_{i+1} \neq q_{i+1}$.

Proof of Claim 2. As $b$ is a permutation of $Q$ and $a$ has rank $n-1$, the transformation given by the word $a b^{n-s}$ has rank $n-1$. So, as precisely one pair is collapsed, we have that if $\left\{p_{i}, q_{i}\right\} \neq\left\{s_{i}, t_{i}\right\}$ and both are 2subsets, then $\left\{p_{i+1}, q_{i+1}\right\} \neq\left\{s_{i+1}, t_{i+1}\right\}$, as only $\left|\left\{p_{i+1}, q_{i+1}, s_{i+1}, t_{i+1}\right\}\right| \in$ $\{2,3,4\}$ is possible because at most one 2 -set could be collapsed. Note that, as by assumption $\left\{p_{0}, q_{0}\right\} \neq\left\{s_{0}, t_{0}\right\}$, this gives inductively $\left\{p_{i}, q_{i}\right\} \neq$ $\left\{s_{i}, t_{i}\right\}$ for all $i$ with $\left|\left\{p_{i}, q_{i}\right\}\right|=\left|\left\{s_{i}, t_{i}\right\}\right|=2$. As $\left|\left\{p_{i}, q_{i}\right\}\right|=\left|\left\{s_{i}, t_{i}\right\}\right|=2$ is assumed in the claim, we have $\left\{p_{i}, q_{i}\right\} \neq\left\{s_{i}, t_{i}\right\}$ and as at most one pair could be collapsed the claim follows.

\footnotetext{
${ }^{6}$ Note that $0<m<n$ implies $\delta\left(q, b^{m}\right) \neq q$. Also note that we added $n$ on the right hand side to account for values $d>1$. In Proposition 10 we only subtract one from the exponent of $b$, which is always non-zero and strictly smaller than $n$, and so we do not needed this "correction for the $b$-cycle" in case of resulting negative exponents.
} 
Claim 3: There exists some $i>0$ such that $p_{i}=q_{i}=q$.

Proof of Claim 3. First, suppose we have some $i \geqslant 0$ and $\left|\left\{p_{i}, q_{i}\right\}\right|=2$ such that, after reading the letter $a$, Equation (2) applies, i.e., we have

$$
\delta\left(q_{i}, a b^{r}\right)=\delta\left(q_{i}, b^{m_{i}} a\right) \text { or } \delta\left(q_{i}, b^{m_{i}} a b^{r}\right)=\delta\left(q_{i}, a\right) .
$$

Write $r=m d$ for some $m>0$.

(i) Suppose $\delta\left(q_{i}, a b^{r}\right)=\delta\left(q_{i}, b^{m_{i}} a\right)=\delta\left(p_{i}, a\right)$.

Then $p_{i+1}=\delta\left(p_{i}, a b^{n-s}\right)=\delta\left(q_{i}, a b^{n-s+r}\right)=\delta\left(q_{i+1}, b^{r}\right)=\delta\left(q_{i}, b^{r}\right)$, using $q_{i}=q$, by Claim 1 , and $\delta\left(q, a b^{n-s}\right)=q$. Then $\delta\left(p_{i+1}, a\right)=$ $\delta\left(q, b^{r} a\right)$. As $r \equiv 0(\bmod n)$, Equation (1) must apply, and, as $0<$ $r<n$, we find

$$
\delta\left(p_{i+1}, a\right)=\delta\left(q, a b^{n+r-d}\right)
$$

which equals $\delta\left(q, a b^{r-d}\right)$ as $r-d \geqslant 0$. So, $p_{i+2}=\delta\left(p_{i+1}, a b^{n-s}\right)=$ $\delta\left(q, a b^{n-s+r-d}\right)=\delta\left(\delta\left(q, a b^{n-s}, b^{r-d}\right)\right)=\delta\left(q, b^{r-d}\right)$. Continuing inductively for $m-1$ additional steps, we find

$$
p_{i+1+m}=\delta\left(q, b^{r-m d}\right)=q=q_{i+1+m} .
$$

(ii) Suppose $\delta\left(q_{i}, b^{m_{i}} a b^{r}\right)=\delta\left(q_{i}, a\right)$.

As $p_{i}=\delta\left(q_{i}, b^{m_{i}}\right)$, we have $\delta\left(p_{i}, a b^{r}\right)=\delta\left(q_{i}, a\right)$. Hence, for $0 \leqslant j<$ $n$ such that $\delta\left(p_{i}, a b^{j}\right)=q$, we have $\delta\left(q_{i}, a b^{j}\right)=\delta\left(\delta\left(p_{i}, a b^{r}\right), b^{j}\right)=$ $\delta\left(\delta\left(p_{i}, a b^{j}\right), b^{r}\right)=\delta\left(q, b^{r}\right)$. Then we can write $r=m d$ and proceed exactly as in Case (i), to find

$$
\delta\left(\left\{q, \delta\left(q, b^{r}\right)\right\},\left(a b^{n-s}\right)^{m}\right)=\{q\}
$$

So that $\left.\left.\delta\left(\left\{\delta\left(p_{i}, a\right), \delta\left(p_{i}, a b^{r}\right)\right)\right\}, b^{j}\left(a b^{n-s}\right)^{m}\right)\right)=\{q\}$.

Otherwise, after reading the letter $a$ in the process of constructing the sequences $p_{i}$ and $q_{i}$, only Equation (1) applies. As $d$ is coprime to $n$, we find $k \geqslant 0$ such that $m-k d \equiv 0(\bmod n)$, i.e., $m-k d+l n=0$ for some $k \geqslant l$ (as $0 \leqslant m<n$, we have $l \leqslant k$, for $l>k$ would imply $l n>k d$, and so we could not have $l n+m=k d$ ). But note that $l \leqslant 0$ is possible. By 
Equation (1) and using $k \geqslant l$,

$$
\begin{array}{rrr}
p_{k}= & \delta\left(p_{0},\left(a b^{n-s}\right)^{k}\right) & \\
= & \delta\left(q_{0}, b^{m}\left(a b^{n-s}\right)^{k}\right) & \\
= & \delta\left(q, b^{m}\left(a b^{n-s}\right)^{k}\right) & \\
= & \delta\left(q, b^{m} a b^{n-s}\left(a b^{m-s}\right)\right] \\
= & \left.\delta\left(q, a b^{n+m-d}\right)^{n-s}\left(a b^{n-s}\right)^{k-1}\right) & \\
= & \delta\left(q, b^{n+m-d}\left(a b^{n-s}\right)^{k-1}\right) & {[\text { Equation }[1]]} \\
= & \delta\left(q, a b^{2 n+m-2 d} b^{n-s}\left(a b^{n-s}\right)^{k-2}\right) & \\
& \vdots & \\
= & \left.\delta\left(q, a b^{n-s}\right)=q\right] \\
= & \delta\left(q, b^{k n+1) n+m-(k-1) d} a b^{n-s}\right) & \\
= & \delta\left(q, b^{k n-l n}\right) \\
= & \delta\left(q, b^{(k-l) n}\right)=q=q_{k} .
\end{array}
$$

So, the word $w=\left(a b^{n-s}\right)^{k}$ maps both states $\left\{p_{0}, q_{0}\right\}$ to the same state $q$.

The Claims (2) and (3) imply that we have some word $w \in\left(a b^{n-s}\right)^{*}$ which collapses precisely one 2-set. For, by Claim (3), we must have some smallest $i>0$ such that $\left|\left\{p_{i-1}, q_{i-1}\right\}\right|=\left|\left\{s_{i-1}, t_{i-1}\right\}\right|=2$ and either $p_{i}=q_{i}$, or $s_{i}=t_{i}$, but not both could be equal by Claim (2) and the minimality of $i$.

Proposition 10. Let $\Sigma=\{a, b\}$ and suppose $\mathscr{A}=(\Sigma, Q, \delta)$ has $n$ states and is completely reachable with the letter a having rank $n-1$ and the letter $b$ permuting the states with a single orbit. Then $\operatorname{sc}(\operatorname{Syn}(\mathscr{A}))=2^{n}-n$ if we can find a state $q \in Q$ such that for all $0 \leqslant m \leqslant\lfloor n / 2\rfloor-1$ we have

$$
\delta\left(q, b^{m+1} a\right)=\delta\left(q, a b^{m}\right) .
$$

Proof. Let $q \in Q$ be the state from the statement. Note that, as $\delta(q, b a)=\delta(q, a)$ and $a$ has rank $n-1$, the letter $a$ acts injective on $Q \backslash\{q\}$. Also, on all states $\delta\left(q, b^{k}\right)$ with $0 \leqslant k \leqslant\lfloor n / 2\rfloor$ the action of $a$ is determined by the state $\delta(q, a)$. We will show that all 2 -sets of states are distinguishable in $\mathcal{P}_{\mathscr{A}}$. By Lemma 2 , this will give our claim. Let $\{s, t\},\{p, r\} \subseteq Q$ be two distinct 2-sets. Choose $m_{1}, m_{2}>0$ minimal such that

$$
\delta\left(s, b^{m_{1}}\right)=t \text { or } \delta\left(t, b^{m_{1}}\right)=s
$$

and

$$
\delta\left(p, b^{m_{2}}\right)=r \text { or } \delta\left(r, b^{m_{2}}\right)=p .
$$

As $\delta\left(t, b^{m_{1}}\right)=s$ if and only if $\delta\left(s, b^{n-m_{1}}\right)=t$, and similarly for $\{p, r\}$, we have $0<m_{1}, m_{2} \leqslant\lfloor n / 2\rfloor$. We will do induction on $\min \left\{m_{1}, m_{2}\right\}$. Without loss of generality, assume $m_{1} \leqslant m_{2}$ and $\delta\left(s, b^{m_{1}}\right)=t$. Choose $0 \leqslant k<n$ such that $\delta\left(s, b^{k}\right)=q$. Then, by assumption, $\delta\left(s, b^{k+m_{1}} a\right)=\delta\left(s, b^{k} a b^{m_{1}-1}\right)$. We distinguish two cases. 
(i) Suppose $s \notin\{p, r\}$. Then $q \notin \delta\left(\{p, r\}, b^{k}\right)$. As $a$ acts injective on $Q \backslash\{q\}$, we have $\left|\delta\left(\{p, r\}, b^{k} a\right)\right|=2$. If $m_{1}=1$, then

$$
\delta\left(s, b^{k} a\right)=\delta\left(s, b^{k} a b^{m_{1}-1}\right)=\delta\left(s, b^{k+m_{1}} a\right)=\delta\left(t, b^{k} a\right) .
$$

Hence $\left|\delta\left(\{s, t\}, b^{k} a\right)\right|=1$ and the sets $\{s, t\}$ and $\{p, r\}$ are distinguished in $\mathcal{P}_{\mathscr{A}}$ by $b^{k} a$. If $m_{1}>1$, then $\delta\left(s, b^{k} a b^{m_{1}-1}\right)=\delta\left(s, b^{k+m_{1}} a\right)=\delta\left(t, b^{k} a\right)$. As $0<m_{1}-1<\lfloor n / 2\rfloor$, we have $\left|\left\{\delta\left(s, b^{k} a\right), \delta\left(t, b^{k} a\right)\right\}\right|=2$. Hence, for the two 2 -sets

$$
\left\{\delta\left(s, b^{k} a\right), \delta\left(t, b^{k} a\right)\right\} \text { and }\left\{\delta\left(p, b^{k} a\right), \delta\left(r, b^{k} a\right)\right\}
$$

the minimal powers of $b$ that map them to each other, i.e., fulfill the corresponding Equations (6) and (7), have strictly smaller exponents than $\min \left\{m_{1}, m_{2}\right\}$. So, we can use our induction hypothesis, implying that some word $u \in \Sigma^{*}$ maps one set to a singleton set, but not the other. Then $b^{k} a u$ would distinguish $\{s, t\}$ and $\{p, r\}$.

(ii) Suppose $s \in\{p, r\}$. Without loss of generality, assume $\delta\left(p, b^{m_{2}}\right)=r$. If $s=p$, then $m_{1}=m_{2}$ would imply $r=t$, which is excluded as both 2-sets are assumed to be distinct. Hence, as $m_{1}=\min \left\{m_{1}, m_{2}\right\}$, we have $m_{1}<m_{2}$. If $m_{1}=1$, then, as in case (i), we have $\left|\delta\left(\{s, t\}, b^{k} a\right)\right|=1$, but

$$
\delta(q, b) \notin\left\{\delta\left(p, b^{k}\right), \delta\left(r, b^{k}\right)\right\} .
$$

As $\delta(q, b)=\delta\left(r, b^{k}\right)$ would imply, as $b$ is a permutation, that $\delta(p, b)=$ $r$. But, as $p=s, \delta\left(p, b^{k}\right)=q$, and $\delta\left(p, b^{m_{2}}\right)=r$ is minimal with $1<$ $m_{2}<\lfloor n / 2\rfloor$. Hence $\delta(p, b)=r$ would contradict the minimality of $m_{2}$. So, as $a$ acts injective on $Q \backslash\{\delta(q, b)\}$, as it only collapses $\{q, \delta(q, b)\}$, we have $\left|\left\{\delta\left(p, b^{k}\right), \delta\left(r, b^{k}\right)\right\}\right|=2$. So, the word $b^{k} a$ distinguishes $\{s, t\}$ and $\{p, r\}$. Now suppose $m_{1}>1$. Then, as in case (i), $\delta\left(s, b^{k} a b^{m_{1}-1}\right)=\delta\left(t, b^{k} a\right)$ and $\left|\left\{\delta\left(s, b^{k} a\right), \delta\left(t, b^{k} a\right)\right\}\right|=2$. Similarly, as $1<m_{2}<n$, we get $\left|\left\{\delta\left(p, b^{k} a\right), \delta\left(r, b^{k} a\right)\right\}\right|=$ 2. But, again, the minimal powers of $b$ that map them to each other, i.e., fulfill the corresponding Equations (6) and (7), have strictly smaller exponents than $\min \left\{m_{1}, m_{2}\right\}$. So, we can use our induction hypothesis, implying that some word $u \in \Sigma^{*}$ maps one set to a singleton set, but not the other. Then $b^{k} a u$ would distinguish $\{s, t\}$ and $\{p, r\}$. If we have $s=r$, then $\delta\left(r, b^{n-m_{2}}\right)=p$. Similarly to the case $s=p$, we have $m_{1}<n-m_{2}$. As $m_{1}<n-m_{2}<n$, we can argue like in the previous case $s=p$ to find either two new 2-sets with a stricly smaller induction parameter, or one is a singleton, but not the other. Note that for $1<m<n$ we have $\left|\delta\left(\left\{q, \delta\left(q, b^{m}\right)\right\}, a\right)\right|=2$.

So, by induction, we can distinguish all 2-sets in $\mathcal{P}_{\mathscr{A}}$.

Some remarks how all the words of rank 3 were generated in Example 1 , to convince the reader that it is indeed correct. I used GAP 7 to compute all words of rank 3 with the following commands.

\footnotetext{
7 https://www.gap-system.org/
} 
gap> LoadPackage ( "SgpViz" ); ;

gap> S:=Semigroup (Transformation $([2,3,4,1])$, Transformation $([2,3,2,4]))$; ;

gap> Print (DotForDrawingDClassOfElement (S,Transformation $([2,3,2,4])))$;

digraph DClassOfElement \{

graph [center=yes, ordering=out];

node [shape=plaintext];

edge [color=cornflowerblue, arrowhead=none];

1 [label=<

$<$ TABLE BORDER="0" CELLBORDER="0" CELLPADDING="0" CELLSPACING="0" PORT="1">

$<$ TR $><$ TD BORDER="0" $><$ TABLE CELLSPACING="0" $><T R>\left\langle T D\right.$ BGCOLOR="white" BORDER="0" $\left.>* b^{\wedge} 2 \mathrm{a}^{\wedge} 2 \mathrm{~b}^{\wedge} 2</ \mathrm{TD}\right\rangle\langle/ \mathrm{TR}\rangle$

$<$ TR $><$ TD BGCOLOR="white" BORDER="O" $\left.>\mathrm{a}^{\wedge} 2 \mathrm{~b}^{\wedge} 2</ \mathrm{TD}\right\rangle\langle/ \mathrm{TR}\rangle$

$<$ TR $><$ TD BGCOLOR="white" BORDER="0" $\left.>\mathrm{ab}^{\wedge} 2 \mathrm{a}^{\wedge} 2 \mathrm{~b}^{\wedge} 2</ \mathrm{TD}\right\rangle\langle/ \mathrm{TR}\rangle$

$<$ TR $><$ TD BGCOLOR="white" BORDER="0" $\left.>\mathrm{ab}^{\wedge} 2 \mathrm{ab}^{\wedge} 2</ \mathrm{TD}\right\rangle\langle/ \mathrm{TR}\rangle$

$\left\langle\right.$ TR $>\left\langle\right.$ TD BGCOLOR="white" BORDER="0" $\left.>\mathrm{ab}^{\wedge} 2</ \mathrm{TD}\right\rangle\langle/ \mathrm{TR}\rangle$

$<$ TR $><$ TD BGCOLOR="white" BORDER="0" $\left.>\mathrm{b}^{\wedge} 2 \mathrm{ab}^{\wedge} 2</ \mathrm{TD}\right\rangle\langle/ \mathrm{TR}>$

$</$ TABLE $></$ TD $><$ TD BORDER="0" $><$ TABLE CELLSPACING="0" $><$ TR $><$ TD BGCOLOR="white" BORDER="0" $>$ a $</$ TD $></$ TR $>$

$\left\langle\right.$ TR $>\left\langle\right.$ TD BGCOLOR="white" BORDER="0" $\left.>\mathrm{b}^{\wedge} 2 \mathrm{a}</ \mathrm{TD}\right\rangle\langle/ \mathrm{TR}\rangle$

$\left\langle\right.$ TR $><$ TD BGCOLOR="white" BORDER="0" $\left.>* \mathrm{a}^{\wedge} 2</ \mathrm{TD}\right\rangle\langle/ \mathrm{TR}\rangle$

$<$ TR $><$ TD BGCOLOR="white" BORDER="O" $\left.>\mathrm{b}^{\wedge} 2 \mathrm{a}^{\wedge} 2</ \mathrm{TD}\right\rangle\langle/ \mathrm{TR}\rangle$

$\left\langle\right.$ TR $>\left\langle\right.$ TD BGCOLOR="white" BORDER="0" $\left.>\mathrm{ab}^{\wedge} 2 \mathrm{a}</ \mathrm{TD}\right\rangle\langle/ \mathrm{TR}\rangle$

$<$ TR $><$ TD BGCOLOR="white" BORDER="0" $\left.>\mathrm{ab}^{\wedge} 2 \mathrm{a}^{\wedge} 2</ \mathrm{TD}\right\rangle\langle/ \mathrm{TR}\rangle$

$</$ TABLE $><$ TD $><$ TD BORDER="0" $><$ TABLE CELLSPACING="0" $>\langle$ TR $><$ TD BGCOLOR="white" BORDER="0" $>$ ab^3</TD $></$ TR $>$

$<$ TR $><$ TD BGCOLOR="white" BORDER="0" $>\mathrm{b}^{\wedge} 2 \mathrm{ab} \wedge 3</ \mathrm{TD}></ \mathrm{TR}>$

$<$ TR $><$ TD BGCOLOR="white" BORDER="0" $\left.>\mathrm{a}^{\wedge} 2 \mathrm{~b}^{\wedge} 3</ \mathrm{TD}\right\rangle\langle/ \mathrm{TR}\rangle$

$<$ TR $>\left\langle\right.$ TD BGCOLOR="white" BORDER="0" $\left.>\mathrm{b}^{\wedge} 2 \mathrm{a}^{\wedge} 2 \mathrm{~b}^{\wedge} 3</ \mathrm{TD}\right\rangle\langle/ \mathrm{TR}\rangle$

$\left\langle\right.$ TR $>\left\langle\right.$ TD BGCOLOR="white" BORDER="0" $\left.>\mathrm{ab}^{\wedge} 2 \mathrm{ab}^{\wedge} 3</ \mathrm{TD}\right\rangle\langle/ \mathrm{TR}\rangle$

$<$ TR $><$ TD BGCOLOR="white" BORDER="0" $\left.>\mathrm{ab}^{\wedge} 2 \mathrm{a}^{\wedge} 2 \mathrm{~b} \wedge 3</ \mathrm{TD}\right\rangle\langle/ \mathrm{TR}\rangle$

$</$ TABLE $><$ TD $><$ TD BORDER="0" $><$ TABLE CELLSPACING="0" $><$ TR $><$ TD BGCOLOR="white" BORDER="0" $>$ ab^ $2 \mathrm{ab}</$ TD $></$ TR $<$ TR $>\left\langle\right.$ TD BGCOLOR="white" BORDER="0" $\left.>\mathrm{ab}^{\wedge} 2 \mathrm{a}^{\wedge} 2 \mathrm{~b}</ \mathrm{TD}\right\rangle\langle/ \mathrm{TR}\rangle$

$\left\langle\right.$ TR $>\left\langle\right.$ TD BGCOLOR="white" BORDER="0" $\left.>\mathrm{b}^{\wedge} 2 \mathrm{ab}</ \mathrm{TD}\right\rangle\langle/ \mathrm{TR}\rangle$

$<$ TR $><$ TD BGCOLOR="white" BORDER="0" $>$ ab $</$ TD $></ T R>$

$\left\langle\right.$ TR $><$ TD BGCOLOR="white" BORDER="0" $\left.>\mathrm{b}^{\wedge} 2 \mathrm{a}^{\wedge} 2 \mathrm{~b}</ \mathrm{TD}\right\rangle\langle/ \mathrm{TR}\rangle$

$\left\langle\right.$ TR $>\left\langle\right.$ TD BGCOLOR="white" BORDER="0" $>\mathrm{a}^{\wedge} 2 \mathrm{~b}\langle/ \mathrm{TD}\rangle\langle/ \mathrm{TR}\rangle$

$</$ TABLE $></$ TD $></ T R>$

$<$ TR $><$ TD BORDER="0" $>\left\langle\right.$ TABLE CELLSPACING="0" $>\left\langle\right.$ TR $>\left\langle\right.$ TD BGCOLOR="white" BORDER="0" $>$ bab^ $\left.2 \mathrm{a}^{\wedge} 2 \mathrm{~b}^{\wedge} 2</ \mathrm{TD}\right\rangle\langle/ \mathrm{TR}\rangle$

$<$ TR $><$ TD BGCOLOR="white" BORDER="0" $>$ bab^2</TD $></ T R>$

$<$ TR $><$ TD BGCOLOR="white" BORDER="0" $>\mathrm{b}^{\wedge} 3 \mathrm{a}^{\wedge} 2 \mathrm{~b}^{\wedge} 2</ \mathrm{TD}>\langle/ \mathrm{TR}\rangle$

$<$ TR $><$ TD BGCOLOR="white" BORDER="0" $>\mathrm{b}^{\wedge} 3 \mathrm{ab} \wedge 2</ \mathrm{TD}></ \mathrm{TR}>$

$<$ TR $><$ TD BGCOLOR="white" BORDER="O" $>$ ba^2 $2 b^{\wedge} 2</$ TD $\rangle\langle/$ TR $>$

$\left\langle\right.$ TR $>\left\langle\right.$ TD BGCOLOR="white" BORDER="0" $\left.>\mathrm{bab}^{\wedge} 2 \mathrm{ab} \wedge 2</ \mathrm{TD}\right\rangle\langle/ \mathrm{TR}\rangle$

$</$ TABLE $><$ TD $><$ TD BORDER="0" $><$ TABLE CELLSPACING="0" $><$ TR $><$ TD BGCOLOR="white" BORDER="0" $>$ ba^ $2</$ TD $></$ TR $>$

$<$ TR $><$ TD BGCOLOR="white" BORDER="0" $>$ bab^2a</TD $></ T R>$

$<$ TR $><$ TD BGCOLOR="white" BORDER="0" $>$ ba $</$ TD $></ T R>$

$\left\langle\right.$ TR $><$ TD BGCOLOR="white" BORDER="0" $\left.>\mathrm{bab}^{\wedge} 2 \mathrm{a}^{\wedge} 2</ \mathrm{TD}\right\rangle\langle/ \mathrm{TR}\rangle$

$<$ TR $><$ TD BGCOLOR="white" BORDER="0" $\left.>\mathrm{b}^{\wedge} 3 \mathrm{a}</ \mathrm{TD}\right\rangle\langle/ \mathrm{TR}\rangle$

$<$ TR $><$ TD BGCOLOR="white" BORDER="0" $>$ b^3a^2</TD $></ T R>$

$</$ TABLE $></$ TD $><$ TD BORDER="0" $><$ TABLE CELLSPACING="0" $><$ TR $><$ TD BGCOLOR="white" BORDER="0" $>*$ ba^2b^3</TD $></$ $<$ TR $><$ TD BGCOLOR="white" BORDER="0" $>$ bab^2ab^3</TD $></ T R>$

$<$ TR $><$ TD BGCOLOR="white" BORDER="0" $>$ bab^3</TD $></ T R>$

$<$ TR $><$ TD BGCOLOR="white" BORDER $=" 0^{\prime \prime}>$ bab^2a^2b^3</TD $></ T R>$

$<$ TR $><$ TD BGCOLOR="white" BORDER="0" $>$ b^3ab^3</TD $></ T R>$

$<$ TR $><$ TD BGCOLOR="white" BORDER="0" $\left.>\mathrm{b}^{\wedge} 3 \mathrm{a}^{\wedge} 2 \mathrm{~b}^{\wedge} 3</ \mathrm{TD}\right\rangle\langle/ \mathrm{TR}\rangle$ 
$</$ TABLE $><$ TD $><$ TD BORDER="0" $><$ TABLE CELLSPACING="0" $><$ TR $><$ TD BGCOLOR="white" BORDER="0" $>$ b^3ab $</$ TD $></$ TR $>$ $\left\langle\right.$ TR $>\left\langle\right.$ TD BGCOLOR="white" BORDER="0" $>* \mathrm{~b}^{\wedge} 3 \mathrm{a}^{\wedge} 2 \mathrm{~b}\langle/ \mathrm{TD}\rangle\langle/ \mathrm{TR}\rangle$

$<$ TR $><$ TD BGCOLOR="white" BORDER="0" $>$ bab^2ab $</$ TD $></ T R>$

$<$ TR $><$ TD BGCOLOR="white" BORDER="0" $>$ ba^2b $</$ TD $></ T R>$

$<$ TR $><$ TD BGCOLOR="white" BORDER="0" $>$ bab^2a^2b $</ T D\rangle\langle/ T R\rangle$

$\langle$ TR $>\langle$ TD BGCOLOR="white" BORDER="0" $>$ bab $\langle/$ TD $\rangle\langle/ T R\rangle$

$</$ TABLE $\rangle\langle/$ TD $\rangle\langle/$ TR $>$

$</$ TABLE $>>$;

\}

gap> Print(DotForDrawingDClassOfElement (S,Transformation $([2,3,2,4])), 1)$;

digraph DClassOfElement \{

graph [center=yes, ordering=out];

node [shape=plaintext];

edge [color=cornflowerblue, arrowhead=none];

$1[$ label $=<$

$<$ TABLE BORDER="0" CELLBORDER="0" CELLPADDING="0" CELLSPACING="0" PORT="1">

$<$ TR $><T D$ BORDER="0" $><$ TABLE CELLSPACING="0" $><T R><T D$ BGCOLOR="white" BORDER="0" $\left.>* b^{\wedge} 2 \mathrm{a}^{\wedge} 2 \mathrm{~b}^{\wedge} 2</ \mathrm{TD}\right\rangle\langle/ \mathrm{TR}\rangle$

$<$ TR $><$ TD BGCOLOR="white" BORDER="0" $\left.>\mathrm{a}^{\wedge} 2 \mathrm{~b}^{\wedge} 2</ \mathrm{TD}\right\rangle\langle/ \mathrm{TR}\rangle$

$<$ TR $><$ TD BGCOLOR="white" BORDER="O" $\left.>\mathrm{ab}^{\wedge} 2 \mathrm{a}^{\wedge} 2 \mathrm{~b}^{\wedge} 2</ \mathrm{TD}\right\rangle\langle/ \mathrm{TR}\rangle$

$<$ TR $><$ TD BGCOLOR="white" BORDER="O" $\left.>\mathrm{ab}^{\wedge} 2 \mathrm{ab}^{\wedge} 2</ \mathrm{TD}\right\rangle\langle/ \mathrm{TR}\rangle$

$<$ TR $><$ TD BGCOLOR="white" BORDER="0" $\left.>\mathrm{ab}^{\wedge} 2</ \mathrm{TD}\right\rangle\langle/ \mathrm{TR}\rangle$

$<$ TR $><$ TD BGCOLOR="white" BORDER="O" $\left.>\mathrm{b}^{\wedge} 2 \mathrm{ab}^{\wedge} 2</ \mathrm{TD}\right\rangle\langle/ \mathrm{TR}\rangle$

$</$ TABLE $></$ TD $><$ TD BORDER="0" $><$ TABLE CELLSPACING="0" $><$ TR $><$ TD BGCOLOR="white" BORDER="0" $>$ a $</$ TD $></$ TR $>$

$<$ TR $><$ TD BGCOLOR="white" BORDER="0" $>\mathrm{b}^{\wedge} 2 \mathrm{a}</ \mathrm{TD}></ \mathrm{TR}>$

$<$ TR $><$ TD BGCOLOR="white" BORDER="0" $>* a^{\wedge} 2</$ TD $></ T R>$

$<$ TR $><$ TD BGCOLOR="white" BORDER="0" $\left.>\mathrm{b}^{\wedge} 2 \mathrm{a}^{\wedge} 2</ \mathrm{TD}\right\rangle\langle/ \mathrm{TR}\rangle$

$\left\langle\right.$ TR $>\left\langle\right.$ TD BGCOLOR="white" BORDER="0" $\left.>\mathrm{ab}^{\wedge} 2 \mathrm{a}</ \mathrm{TD}\right\rangle\langle/ \mathrm{TR}\rangle$

$<$ TR $><$ TD BGCOLOR="white" BORDER="0" $\left.>\mathrm{ab}^{\wedge} 2 \mathrm{a}^{\wedge} 2</ \mathrm{TD}\right\rangle\langle/ \mathrm{TR}\rangle$

$</$ TABLE $></$ TD $><$ TD BORDER="0" $><$ TABLE CELLSPACING="0" $><$ TR $><$ TD BGCOLOR="white" BORDER="0" $>$ ab^3</TD $></$ TR $>$

$\left\langle\right.$ TR $><$ TD BGCOLOR="white" BORDER="O" $\left.>\mathrm{b}^{\wedge} 2 \mathrm{ab} \wedge 3</ \mathrm{TD}\right\rangle\langle/ \mathrm{TR}\rangle$

$<$ TR $><$ TD BGCOLOR="white" BORDER $=" 0 ">a^{\wedge} 2 b^{\wedge} 3</$ TD $></ T R>$

$<$ TR $><$ TD BGCOLOR="white" BORDER="O" $\left.>\mathrm{b}^{\wedge} 2 \mathrm{a}^{\wedge} 2 \mathrm{~b}^{\wedge} 3</ \mathrm{TD}\right\rangle\langle/ \mathrm{TR}\rangle$

$<$ TR $><$ TD BGCOLOR="white" BORDER="0" $\left.>\mathrm{ab}^{\wedge} 2 \mathrm{ab} \leadsto 3</ \mathrm{TD}\right\rangle\langle/ \mathrm{TR}\rangle$

$<$ TR $><$ TD BGCOLOR="white" BORDER="O" $>\mathrm{ab}^{\wedge} 2 \mathrm{a}^{\wedge} 2 \mathrm{~b}^{\wedge} 3</ \mathrm{TD}>\langle/ \mathrm{TR}\rangle$

$</$ TABLE $><$ TD $><$ TD BORDER="0" $><$ TABLE CELLSPACING="0" $><$ TR $><$ TD BGCOLOR="white" BORDER="0" $>$ ab^2ab $</$ TD $></$ TR $<$ TR $><$ TD BGCOLOR="white" BORDER="O" $\left.>\mathrm{ab}^{\wedge} 2 \mathrm{a}^{\wedge} 2 \mathrm{~b}</ \mathrm{TD}\right\rangle\langle/ \mathrm{TR}\rangle$

$<$ TR $><$ TD BGCOLOR="white" BORDER="0" $\left.>\mathrm{b}^{\wedge} 2 \mathrm{ab}</ \mathrm{TD}\right\rangle\langle/ \mathrm{TR}\rangle$

$<$ TR $><$ TD BGCOLOR="white" BORDER="0" $>$ ab $</$ TD $></ T R>$

$<$ TR $><$ TD BGCOLOR="white" BORDER="0" $>\mathrm{b}^{\wedge} 2 \mathrm{a}^{\wedge} 2 \mathrm{~b}</ \mathrm{TD}>\langle/ \mathrm{TR}>$

$<$ TR $><$ TD BGCOLOR="white" BORDER="O" $\left.>\mathrm{a}^{\wedge} 2 \mathrm{~b}</ \mathrm{TD}\right\rangle\langle/ \mathrm{TR}\rangle$

$\langle/$ TABLE $\rangle\langle/$ TD $\rangle\langle/$ TR $>$

$<$ TR $><$ TD BORDER="0" $><$ TABLE CELLSPACING="0" $><$ TR $><$ TD BGCOLOR="white" BORDER="0" $>$ bab^2a^2b^2</TD $></ T R>$

$<$ TR $><$ TD BGCOLOR="white" BORDER="0" $>$ bab^ $2</$ TD $\rangle\langle/$ TR $>$

$<$ TR $><$ TD BGCOLOR="white" BORDER="O" $\left.>\mathrm{b}^{\wedge} 3 \mathrm{a}^{\wedge} 2 \mathrm{~b}^{\wedge} 2</ \mathrm{TD}\right\rangle\langle/ \mathrm{TR}\rangle$

$<$ TR $><$ TD BGCOLOR="white" BORDER="0" $\left.>\mathrm{b}^{\wedge} 3 \mathrm{ab}^{\wedge} 2</ \mathrm{TD}\right\rangle\langle/ \mathrm{TR}\rangle$

$<$ TR $><$ TD BGCOLOR="white" BORDER="0" $\left.>\mathrm{ba}^{\wedge} 2 \mathrm{~b}^{\wedge} 2</ \mathrm{TD}\right\rangle\langle/ \mathrm{TR}\rangle$

$<$ TR $><$ TD BGCOLOR="white" BORDER="0" $>$ bab^2ab^2</TD $></ T R>$

$</$ TABLE $></$ TD $><$ TD BORDER="0" $><$ TABLE CELLSPACING="0" $><$ TR $><$ TD BGCOLOR="white" BORDER="0" $>$ ba^2 $2</$ TD $></$ TR $>$

$<\mathrm{TR}>\langle$ TD BGCOLOR="white" BORDER="0" $>$ bab^2a $</ \mathrm{TD}\rangle\langle/ \mathrm{TR}\rangle$

$<$ TR $><$ TD BGCOLOR="white" BORDER="0" $>$ ba $</$ TD $></ T R>$

$<$ TR $><$ TD BGCOLOR="white" BORDER="0" $>$ bab $\left.^{\wedge} 2 \mathrm{a}^{\wedge} 2</ \mathrm{TD}\right\rangle\langle/ \mathrm{TR}\rangle$

$<\mathrm{TR}><\mathrm{TD}$ BGCOLOR="white" BORDER="0" $>\mathrm{b}$ ^3a $</ \mathrm{TD}\rangle\langle/ \mathrm{TR}\rangle$ 
$<$ TR $><$ TD BGCOLOR="white" BORDER="0" $\left.>\mathrm{b}^{\wedge} 3 \mathrm{a}^{\wedge} 2</ \mathrm{TD}\right\rangle\langle/ \mathrm{TR}\rangle$

$\langle/$ TABLE $\rangle\langle/$ TD $\rangle\left\langle\right.$ TD BORDER="0" $>\langle$ TABLE CELLSPACING="0" $\rangle\langle$ TR $\rangle\left\langle\right.$ TD BGCOLOR="white" BORDER="0" $>*$ ba^ $2 b^{\wedge} 3</$ TD $\rangle\langle/ "$ $<$ TR $><$ TD BGCOLOR="white" BORDER="O" $>$ bab^2ab^3</TD $></ T R>$

$<$ TR $><$ TD BGCOLOR="white" BORDER="0" $>$ bab^3</TD $></$ TR $>$

$<$ TR $><$ TD BGCOLOR="white" BORDER="0" $>$ bab^2a^2b^3</TD $></ T R>$

$<$ TR $><$ TD BGCOLOR="white" BORDER="0" $>b^{\wedge} 3 a a^{\wedge} 3</ T D></ T R>$

$<$ TR $><$ TD BGCOLOR="white" BORDER="0" $\left.>\mathrm{b}^{\wedge} 3 \mathrm{a} \mathrm{a}^{\wedge} 2 \mathrm{~b} \wedge 3</ \mathrm{TD}\right\rangle\langle/ \mathrm{TR}\rangle$

$</$ TABLE $><$ TD $><$ TD BORDER="0" $><$ TABLE CELLSPACING="0" $><$ TR $><$ TD BGCOLOR="white" BORDER="0" $>$ b^3ab $</$ TD $></$ TR $>$

$<$ TR $><$ TD BGCOLOR="white" BORDER="0" $\left.>* \mathrm{~b}^{\wedge} 3 \mathrm{a}^{\wedge} 2 \mathrm{~b}</ \mathrm{TD}\right\rangle\langle/ \mathrm{TR}\rangle$

$<$ TR $><$ TD BGCOLOR="white" BORDER="0" $>$ bab^2ab $</$ TD $>\langle/ T R>$

$<$ TR $><$ TD BGCOLOR="white" BORDER="0" $>$ ba^2b $</$ TD $></ T R>$

$<$ TR $><$ TD BGCOLOR="white" BORDER="0" $>$ bab^ $\left.2 \mathrm{a}^{\wedge} 2 \mathrm{~b}</ \mathrm{TD}\right\rangle\langle/ \mathrm{TR}\rangle$

$<$ TR $><$ TD BGCOLOR="white" BORDER="0" $>$ bab $</$ TD $\rangle\langle/$ TR $>$

$</$ TABLE $></$ TD $></$ TR $>$

$</$ TABLE $>>$ ] ;

\}

1

This output could be rendered with GRAPHIVI $2^{8}$ by the following commands: dot -Tpng <inputfile> -o <outputfile>, which will produce png files as output. Note that, if used on Windows, after installation, the command dot -c has to be executed to install additional plugins, for example for rendering. If everything went fine, the following output should be produced.

\begin{tabular}{|l|l|l|l|}
\hline$[1,2,1,4]$ & {$[2,3,2,4]$} & {$[1,2,1,3]$} & {$[1,3,1,4]$} \\
{$[1,4,1,2]$} & {$[2,4,2,3]$} & {$[1,3,1,2]$} & {$[1,4,1,3]$} \\
{$[2,1,2,4]$} & $*[3,2,3,4]$ & {$[2,1,2,3]$} & {$[3,1,3,4]$} \\
{$[2,4,2,1]$} & {$[3,4,3,2]$} & {$[2,3,2,1]$} & {$[3,4,3,1]$} \\
{$[4,1,4,2]$} & {$[4,2,4,3]$} & {$[3,1,3,2]$} & {$[4,1,4,3]$} \\
{$[4,2,4,1]$} & {$[4,3,4,2]$} & {$[3,2,3,1]$} & {$[4,3,4,1]$} \\
\hline$[1,2,4,2]$ & {$[2,3,4,3]$} & $*[1,2,3,2]$ & {$[1,3,4,3]$} \\
{$[1,4,2,4]$} & {$[2,4,3,4]$} & {$[1,3,2,3]$} & $*[1,4,3,4]$ \\
{$[2,1,4,1]$} & {$[3,2,4,2]$} & {$[2,1,3,1]$} & {$[3,1,4,1]$} \\
{$[2,4,1,4]$} & {$[3,4,2,4]$} & {$[2,3,1,3]$} & {$[3,4,1,4]$} \\
{$[4,1,2,1]$} & {$[4,2,3,2]$} & {$[3,1,2,1]$} & {$[4,1,3,1]$} \\
{$[4,2,1,2]$} & {$[4,3,2,3]$} & {$[3,2,1,2]$} & {$[4,3,1,3]$} \\
\hline
\end{tabular}

\begin{tabular}{|c|c|c|c|}
\hline $\begin{array}{c}{ }^{*} b^{\wedge} 2 a^{\wedge} 2 b^{\wedge} 2 \\
a^{\wedge} 2 b^{\wedge} 2 \\
a b^{\wedge} 2 a^{\wedge} 2 b^{\wedge} 2 \\
a b^{\wedge} 2 a b^{\wedge} 2 \\
a b^{\wedge} 2 \\
b^{\wedge} 2 a b^{\wedge} 2\end{array}$ & $\begin{array}{c}a \\
b^{\wedge} 2 a \\
* a^{\wedge} 2 \\
b^{\wedge} 2 a^{\wedge} 2 \\
a b^{\wedge} 2 a \\
a^{\wedge} / 2 a^{\wedge} 2\end{array}$ & $\begin{array}{c}a b^{\wedge} 3 \\
b^{\wedge} 2 b^{\wedge} 3 \\
a^{\wedge} 2 b^{\wedge} 3 \\
b^{\wedge} 2 a^{\wedge} 2 b^{\wedge} 3 \\
a b^{\wedge} 22 b^{\wedge} 3 \\
a b^{\wedge} 2 a^{\wedge} 2 b^{\wedge} 3\end{array}$ & $\begin{array}{c}a b^{\wedge} 2 a b \\
a b^{\wedge} 2 a^{\wedge} 2 b \\
b^{\wedge} 2 a b \\
a b \\
b^{\wedge} 2 a^{\wedge} 2 b \\
a^{\wedge} 2 b\end{array}$ \\
\hline $\mathrm{bab}^{\wedge} 2 \mathrm{a}^{\wedge} 2 \mathrm{~b}^{\wedge} 2$ & $\mathrm{ba}^{\wedge} 2$ & *ba $\mathbf{a}^{\prime} 2 \mathbf{b}^{\wedge} 3$ & $b^{\prime} 3 a b$ \\
\hline $\mathrm{bab} / 2$ & $\mathrm{bab}^{\wedge} 2 \mathrm{a}$ & $\mathrm{bab}^{\wedge} 2 \mathrm{ab}^{\wedge} 3$ & $* b^{\wedge} 3 a^{\wedge} 2 b$ \\
\hline$b^{\wedge} 3 a^{\wedge} 2 b^{\wedge} 2$ & ba & $\mathrm{bab}^{\wedge} 3$ & $b a b^{\wedge} 2 a b$ \\
\hline$b^{\wedge} 3 a^{\prime} / 2$ & $\mathrm{bab}^{\wedge} 2 \mathrm{a}^{\wedge} 2$ & $b a b^{\wedge} 2 a^{\wedge} 2 b^{\wedge} 3$ & $b^{\wedge} 2 b$ \\
\hline$b^{\wedge} 2 b^{\wedge} 2$ & $b^{\wedge} 3 a$ & $b^{\wedge} 3 a b^{\wedge 3}$ & $b a b^{\wedge} 2 a^{\wedge} 2 b$ \\
\hline $\mathrm{bab}^{\wedge} / 2 \mathrm{ab}^{\wedge} / 2$ & $b^{\wedge} 3 a^{\wedge} 2$ & $b^{\wedge} 3 a^{\wedge} 2 b^{\wedge} 3$ & bab \\
\hline
\end{tabular}

\footnotetext{
8 https://graphviz.org/
} 